\title{
Utilisations villageoises et potentialités technologiques des bois de forêts secondaires dans le Menabe central,
}

Tahiana RAMANANANTOANDRO $\mathbf{1}^{\mathbf{1 ,}}$

Christophe BeLLONCLE ${ }^{2}$

Gabrielle L. RAJOELISON ${ }^{1}$

Jean-Pierre SORG ${ }^{3}$

1 Université d'Antananarivo

École supérieure des sciences

agronomiques

Département des eaux et forêts

BP 175, Antananarivo 101

Madagascar

2 LUNAM Université

École supérieure du bois

Rue Christian Pauc

BP 10605

44306 Nantes

France

${ }^{3}$ École polytechnique fédérale de Zurich Groupe de foresterie pour le développement

ETH Zurich, CHN F75.2

Universtätstrasse 16

8092 Zurich

Suisse

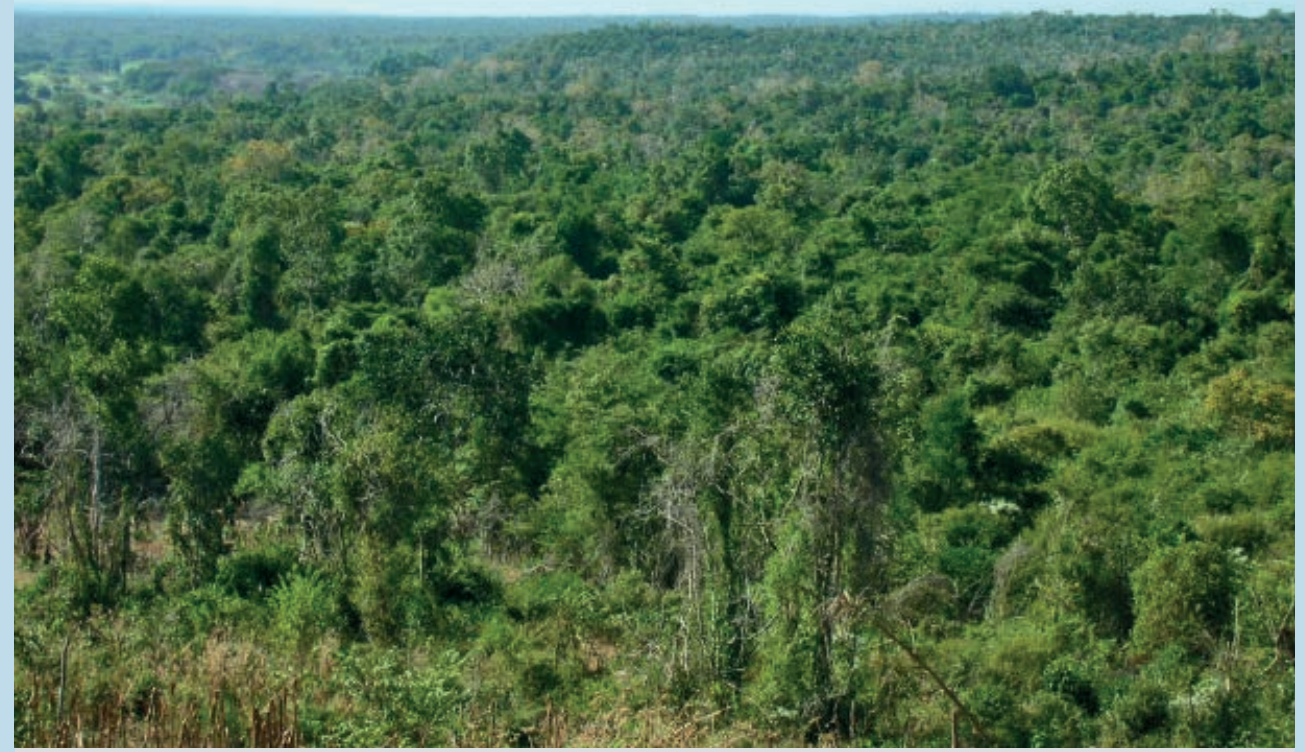

Photo 1.

Forêt primaire dans le Menabe central, actuellement intégrée dans un système

de gestion plus stricte par la mise en place d'une aire protégée.

Photo V. Razafintsalama. 


\section{RÉSUMÉ}

\section{UTILISATIONS VILLAGEOISES ET POTENTIALITÉS TECHNOLOGIQUES DES BOIS DE FORÊTS SECONDAIRES DANS LE MENABE CENTRAL, MADAGASCAR}

Les forêts secondaires sont actuellement des composantes importantes de nombreux paysages forestiers. Dans le Menabe central, au Sud-Ouest de Madagascar, la valorisation des forêts secondaires permet de mieux assurer le maintien des forêts primaires qui ont tendance à se raréfier et qui sont actuellement intégrées dans un système de gestion plus stricte à travers la mise en place d'une aire protégée. Cette étude a été menée pour mieux connaître les utilisations villageoises et les potentialités des forêts secondaires dans la région du Menabe. L'approche socio-économique a permis d'identifier trois types d'utilisations des bois prélevés dans les forêts secondaires : les constructions permanentes, les constructions légères et les sources d'énergie. Trois espèces de forêts secondaires ont été identifiées comme prioritaires dans les choix des villageois : Rhopalocarpus lucidus, Ziziphus mauritiana et Grewia picta. L'analyse des caractères physico-mécaniques des bois de ces trois espèces a mis en évidence des propriétés technologiques intéressantes, comparables aux quatre espèces de référence devenues rares ou absentes dans leur zone de prélèvement : Hernandia voyroni, Dalbergia spp., Cedrelopsis grevei, Commiphora mafaiboa. Ces forêts secondaires méritent désormais d'être prises en compte et valorisées dans le cadre des politiques de gestion forestière au niveau national et international pour assurer leur durabilité.

Mots-clés : bois, forêts secondaires, communautés villageoises, propriétés technologiques, espèces alternatives, Menabe central, Madagascar.

\section{ABSTRACT}

\section{VILLAGE USES AND TECHNOLOGICAL POTENTIAL OF TIMBER FROM SECONDARY FORESTS IN MADAGASCAR'S CENTRAL MENABE REGION}

Secondary forests have become a major feature of many forest landscapes. In the central Menabe region of south-western Madagascar, the use of secondary forests is helping to preserve primary forests, which are becoming scarcer and are now subject to stricter management rules as protected areas. This study was conducted to find out more about village uses of secondary forests in the Menabe region, and their potential. A socio-economic study identified three main uses of timber extracted from secondary forests: for permanent construction, for light construction and as fuel. It has been found that the villagers gave priority to three species of secondary forests Rhopalocarpus lucidus, Ziziphus mauritiana and Grewia picta. An analysis of the physical and mechanical properties of these three species showed technological potential comparable to that of four timber species that are now rarely found, if at all, in the zones where they are extracted: Hernandia voyroni, Dalbergia spp., Cedrelopsis grevei, Commiphora mafaiboa. Economic uses of these scondary forests deserve consideration in the development of national and international forest policies, to ensure the conservation of their resources.

Keywords: wood, secondary forests, village commnities, technological properties, alternative species, central Menabe, Madagascar

\section{USOS CAMPESINOS Y POTENCIALIDADES TECNOLÓGICAS DE MADERAS DE BOSQUES SECUNDARIOS EN MENABE CENTRAL, MADAGASCAR}

Actualmente los bosques secundarios son importantes componentes de numerosos paisajes forestales. En la región del Menabe Central, en el sudoeste de Madagascar, la valorización de los bosques secundarios permite garantizar mejor el mantenimiento de los bosques primarios, que tienen tendencia a enrarecerse y que en la actualidad están integrados en un sistema de manejo más estricto mediante el establecimiento de un área protegida. Este estudio se realizó para conocer mejor los usos campesinos y las potencialidades de los bosques secundarios en la región de Menabe. El enfoque socioeconómico permitió identificar tres tipos de utilización de las maderas extraídas en los bosques secundarios: construcciones permanentes, construcciones ligeras y fuentes de energía. Se identificaron tres especies de los bosques secundarios prioritarias en la elección de los campesinos: Rhopalocarpus lucidus, Ziziphus mauritiana y Grewia picta. El análisis de los caracteres físicomecánicos de la madera de estas tres especies puso de manifiesto unas interesantes propiedades tecnológicas, comparables a las cuatro especies de referencia que se han vuelto escasas o están ausentes en su zona de recolección: Hernandia voyroni, Dalbergia spp., Cedrelopsis greve y Commiphora mafaiboa. A partir de ahora, estos bosques secundarios merecen que se tomen en consideración y se valoricen en el marco de políticas de manejo forestal, a nivel nacional e internacional, para garantizar su sostenibilidad.

Palabras clave: madera, bosques secundarios, comunidades campesinas, propiedades tecnológicas, especies alternativas, Menabe Central, Madagascar. 


\section{INTRODUCTION}

La forêt a toujours occupé une place importante dans la satisfaction des besoins de l'homme en matière de ressources et d'espaces. À Madagascar (DGEF, 2003) comme dans d'autres pays en Afrique (LUBINI, 2003), en Amérique latine (DE LAS SALAS, 2002) ou en Asie (EMRICH et al., 2000), les utilisations des produits et services des forêts par la population conduisent à la disparition progressive des forêts primaires. Dans la région du Menabe, où la végétation caractéristique est la forêt dense sèche, la superficie de la forêt naturelle a été estimée à 958800 hectares en 1900 , contre 879300 hectares en 2005 , soit une perte de $8,3 \%$ en un siècle (MEFT et al., 2009). Cette perte est attribuée en grande partie aux défrichements agricoles et pastoraux, ainsi qu'à l'exploitation du bois pour les diverses utilisations aussi bien locales, régionales que nationales, voire internationales. En raison de la disparition de la forêt primaire (photo 1), les forêts secondaires (photo 2) constituent un élément majeur du paysage forestier de la région. Dans le cadre de cette étude, les forêts secondaires sont définies comme " des forêts qui résultent de la régénération naturelle des forêts défrichées en vue de l'agriculture ou de l'élevage, des forêts exploitées et ayant un âge d'évolution entre une et cinquante années " (FAO, 2003). Les forêts qui ont plus de cinquante ans sont considérées comme des forêts matures peu ou pas perturbées qui peuvent tendre vers la reconstitution naturelle des caractéristiques des formations originelles. Les forêts secondaires sont, en fait, des systèmes dynamiques en succession évoluant vers des forêts qui tendent à se stabiliser. Elles font partie d'une série évolutive dont le développement peut aller dans différentes directions selon les perturbations ou les interventions. Ces formations forestières sont perçues comme des forêts de qualité inférieure alors que les communautés humaines qui vivent à proximité peuvent grandement profiter de leur existence. En effet, la valorisation des forêts secondaires doit permettre de pallier la forte dépendance aux forêts primaires qui sont soumises à un système de gestion plus strict au sein d'aires protégées. En outre, plusieurs espèces endémiques de valeur et utiles telles que Hernandia voyroni, Dalbergia spp., Cedrelopsis grevei et Commiphora mafaiboa se raréfient suite à la déforestation et aux exploitations sélectives et massives ciblant ces espèces (RATSINJOMANANA, 2006 ; ANDRIAMBELO, 2010). Face à la raréfaction de ces espèces de forêts primaires, aussi bien dans les écosystèmes résiduels que sur le marché du bois, la recherche d'espèces alternatives constitue une des solutions envisageables. Ce sont les forêts secondaires qui peuvent fournir ces espèces de substitution et le recours à ces forêts s'avère d'autant plus nécessaire qu'elles constituent les écosystèmes forestiers d'avenir les plus importants en superficie dans le Menabe central ; cela met également en jeu la capacité d'adaptation des villageois aux changements des ressources naturelles, aussi bien qualitativement que quantitativement.

De nombreuses recherches ont été entreprises dans le Menabe sur la préservation et l'aménagement durable de la forêt naturelle (SORG et al., 2003 ; DIRAC RAMOHAVELO, 2009 ; ANDRIAMBELO, 2010). Il en ressort que, contrairement aux forêts primaires, les connaissances sur les forêts secondaires du Menabe ne sont pas très avancées. Dans ce contexte, cette étude a été menée afin de connaître les possibilités d'utilisation des bois issus des forêts secondaires dont il s'agit d'identifier les espèces les plus importantes pour les villageois et d'en connaître la valeur technologique. Pour ce faire, les différentes catégories d'utilisation des bois, dont les demandes peuvent être assurées par les forêts secondaires, ont été identifiées et, pour chaque catégorie, les essences les plus prisées par les villageois ainsi que leurs critères de choix ont été analysés. Enfin, une étude exploratoire a été menée pour savoir si les caractéristiques physico-mécaniques de ces espèces, dites secondaires, permettraient de subvenir aux besoins de fourniture de matériau bois et si ces essences pourraient ainsi se substituer à celles issues des forêts primaires.

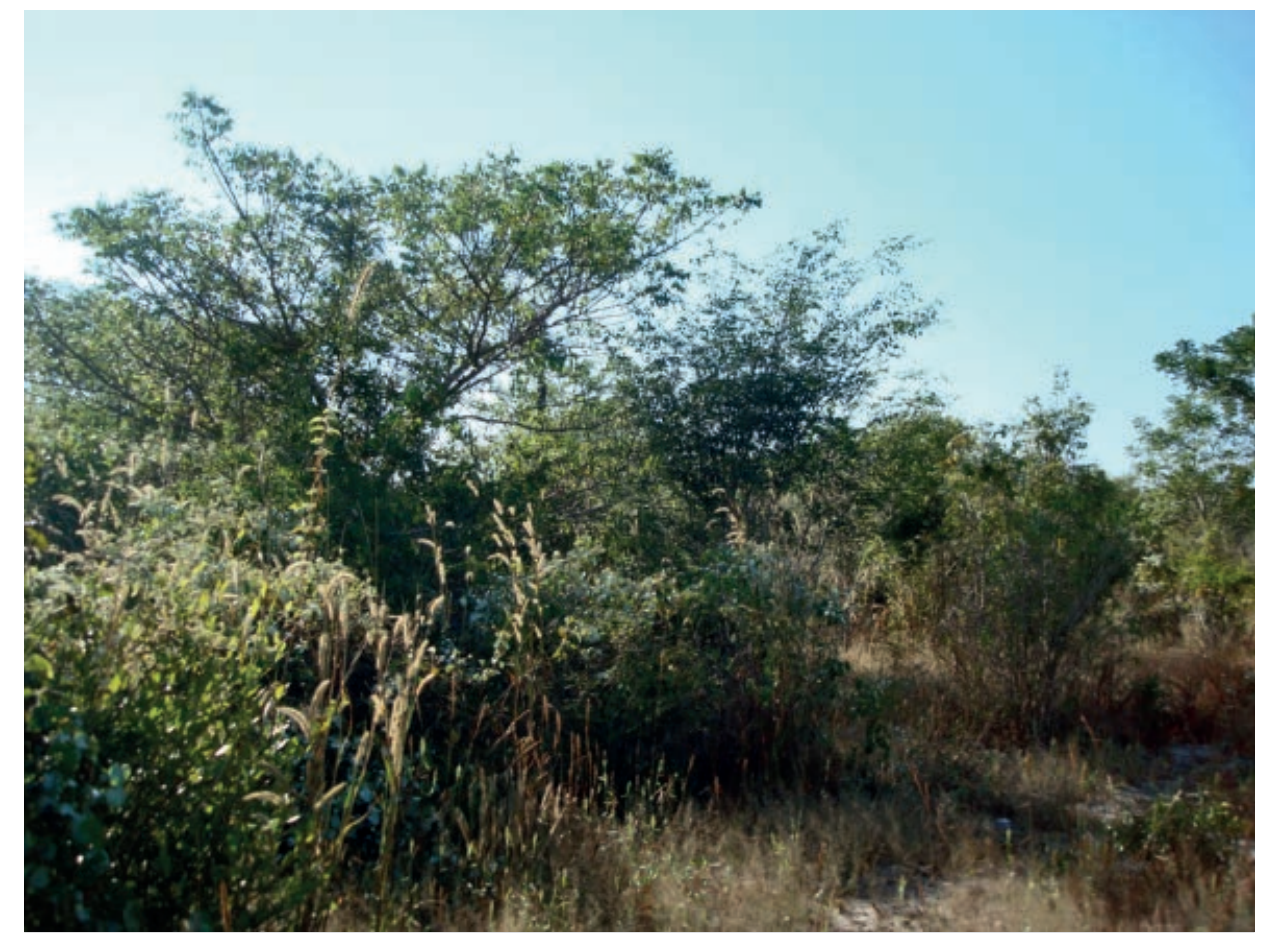

Photo 2.

Forêt secondaire ( 5 ans), élément majeur du paysage forestier dans le Menabe central. Photo V. Razafintsalama. 


\section{Matériels et méthodes}

\section{Zones d'étude et cadre physique}

Le Menabe est une des vingt-deux régions de Madagascar, située le long de la côte Ouest. Il côtoie le canal de Mozambique sur $350 \mathrm{~km}$ de long pour une superficie totale de $48860 \mathrm{~km}^{2}$. Le Menabe central auquel se réfère cet article est une partie de la région du Menabe et est localisé géographiquement entre la rivière Andranomena au Sud et le fleuve Tsiribihina au Nord. L'Ouest est délimité par le canal de Mozambique. À l’Est, la frontière mal définie se termine avec la forêt dense sèche.

Le Menabe jouit d'un climat tropical chaud et subaride (KOECHLIN et al., 1997) avec huit mois écologiquement secs, d'avril à novembre, pendant lesquels la pluviosité est inférieure à $50 \mathrm{~mm}$ et quatre mois de saison chaude et pluvieuse, de décembre à mars, avec des précipitations dépassant $100 \mathrm{~mm}$ par mois, en moyenne. Les extrêmes pluvieux sont souvent liés au passage de dépressions tropicales et cyclones (ANDRIAMBELO, 2010). Le Menabe appartient géologiquement aux formations sédimentaires dues aux dépôts pliocènes continentaux, recouverts d'une carapace argilosableuse appelée aussi sables roux (BOURGEAT et al., 1995). Les formations pédologiques se caractérisent par une prédominance très nette de sols ferrugineux tropicaux sur l'ensemble de la région, avec, en bordure du littoral du canal de Mozambique, des sols halomorphes et des sols minéraux bruts sur la côte Est de Bemaraha (MAEP, 2003). La végétation forestière du Menabe est une forêt dense sèche caducifoliée ou tropophile de l'Ouest (MOAT, SMITH, 2007). C'est un peuplement fermé pluristrate, de stature moins élevée que la forêt dense humide; la plupart des arbres des étages supérieurs perdent leurs feuilles, le sousbois arbustif est soit sempervirent, soit décidu et le tapis graminéen est généralement discontinu (GUILLAUMET, KOECHLIN, 1971), voire inexistant. Pour passer la longue saison sèche, les végétaux ont développé de nombreuses formes et mécanismes d'adaptation à l'aridité, notamment la réduction de la surface foliaire (caducifolie et microphyllie), le développement d'organes de stockage d'eau dans les tiges (pachycaulie) ou dans les feuilles (crassulescence).

\section{Milieu humain et choix des terroirs d'intervention}

La population totale de la région du Menabe a été estimée à 300000 habitants en 2003 (MAEP, 2003), soit une densité de 6,2 habitants $/ \mathrm{km}^{2}$. Cette population est inégalement répartie dans le milieu rural et le milieu urbain avec un taux moyen plus important en milieu rural (soit 75,5\% contre $24,5 \%$ ). C'est une population jeune dont l'âge moyen est de 23 ans. Les personnes d'âge économiquement actif (15 à 64 ans) constituent plus de la moitié de la population et forment une ressource humaine non négligeable pour l'essor de l'économie de la région, notamment en milieu rural. Concernant la répartition des sexes, un certain équilibre est observé avec cependant un déficit dans la classe d'âge de 10 à 14 ans contre un excédent dans la classe d'âge de 15 à 19 ans pour les femmes (MAEP, 2003).

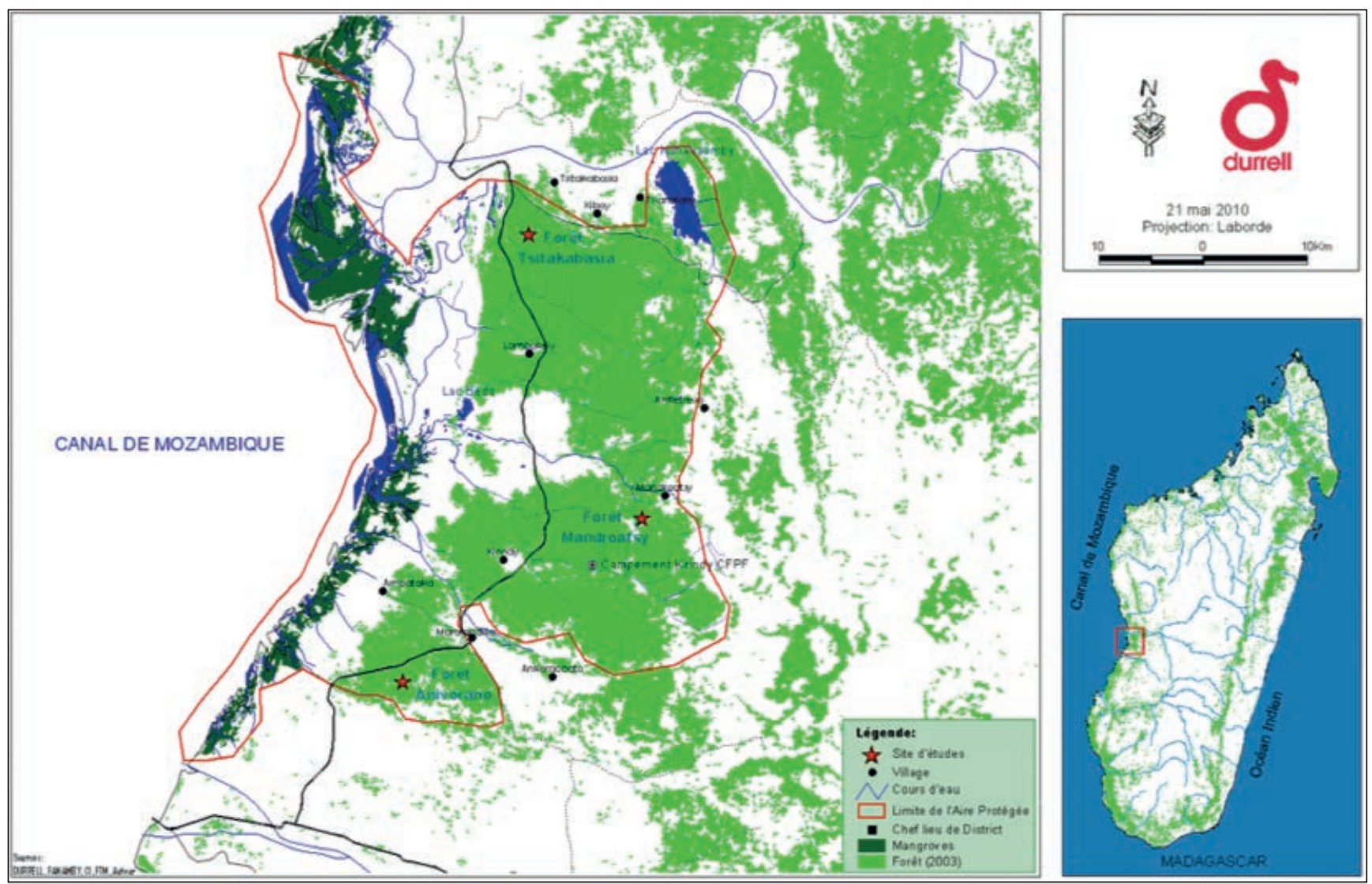

Figure 1.

Localisation des sites d'étude. 
Le Menabe est un terroir sakalava, composé à l'origine par les Sakalava Masikoro (à l'intérieur) et les Sakalava Vezo (sur le littoral). Néanmoins, depuis plus d'un siècle, c'est une terre d'accueil pour des migrants en provenance d'autres régions de Madagascar ou même de l'extérieur (RANDRIAMIDONA, 1990 ; FAUROUX, 2001). Des flux migratoires vers le Menabe se sont opérés depuis les Hautes Terres de Madagascar (Betsileo), le Sud-Est (Korao) et l'extrême Sud (Antandroy). Ainsi, les migrants betsileo ont commencé à développer des systèmes de production fondés sur la riziculture irriguée, ceux du Sud-Est se sont intéressés aux cultures de décrue sur baiboho. Par contre, les Antandroy se sont limités à l'essartage sur brûlis forestiers, une de leurs spécialités (FAUROUX, 2001). Ces migrations dans le Menabe ont ainsi apporté des modifications dans la composition ethnique de la région en général et particulièrement leur répartition dans les terroirs. C'est ainsi que des villages sont caractérisés et façonnés par une ethnie majoritaire. En corollaire, le système de production régional et les formes d'utilisation des ressources forestières se trouvent aussi changés. En effet, une disparité de points de vue est observée selon les groupes ethniques concernant les rôles des forêts dans leur vie quotidienne (CABALZAR, 1996). Les Sakalava, qui sont l'ethnie autochtone, considèrent la forêt comme une réserve de bois pour les divers cultes (cercueils, talismans...) et parfois comme une source de bois de service et de construction. Comme ce groupe ethnique est traditionnellement éleveur, la forêt est aussi utilisée comme terrain de pâturage durant la saison sèche. Pour les immigrants du Sud-Est, plus habitués à pratiquer des cultures dans les plaines alluviales, les forêts constituent des suppléments de terrain pour d'éventuelles cultures pluviales. La considération des Antandroy pour la forêt est surtout dictée par la conquête de terrains de culture. Ainsi, pour eux, la forêt est vouée aux défrichements. Il est à noter que les Betsileo figurent aussi parmi les groupes ethniques d'immigrants dans le Menabe. Néanmoins, ils ne sont pas présents en majorité dans un terroir villageois.

Trois terroirs villageois du Menabe central ont été choisis pour mener cette recherche : Tsitakabasia, Mandroatsy et Anivorano. La raison principale du choix de ces villages repose sur leur emplacement géographique. En effet, ils se situent respectivement au nord, au centre et au sud de l'aire protégée en création dans le Menabe central, en particulier le noyau dur (figure 1). De plus, ils sont caractérisés par des groupes ethniques majoritaires (tableau I).
Les forêts de Tsitakabasia et de Mandroatsy sont en mode de gestion contractualisée entre le service forestier et les communautés de base. La forêt villageoise de Mandroatsy côtoie la zone de conservation prioritaire de la nouvelle aire protégée en création et se trouve concernée aussi par les mesures de contrôle de cette zone. Dans le terroir d'Anivorano, la population n'utilise que des forêts secondaires qui ne sont régies par aucune règle de gestion écrite. La forêt de ce village côtoie, cependant, la Réserve spéciale d’Andranomena gérée par Madagascar National Parks. Le pourcentage des principales ethnies dans chaque village a été calculé à partir des données de recensement de la population obtenues auprès des chefs de fokontany en 2008.

\section{Identification des essences importantes pour les villageois}

En se basant sur les principes du Participatory Rural Appraisal (CHAMBERS, 1994), des observations directes et des enquêtes ont été effectuées dans les trois terroirs villageois. La première étape a consisté à organiser une réunion avec les responsables des villages afin d'expliquer les objectifs de la recherche et de demander la participation des villageois dans sa réalisation. Par la suite, des séjours alternés dans les trois terroirs ont été effectués pour la collecte de données proprement dite. Chaque village a été visité quatre fois pour une durée de 12 jours par visite.

Les observations directes ont été faites dans le but d'avoir un aperçu global du terroir visité. Des parcours dans le village ont été ainsi effectués sans poser de questions aux villageois. Ils ont permis de mieux connaître les activités des villageois, la structure de la société et le mode de vie en général. Ces observations directes ont constitué également des moyens de recoupement des réponses obtenues après enquête.

Trois techniques d'enquête ont été utilisées suivant les objectifs visés et les personnes enquêtées. Il y a d'abord les enquêtes par questionnaire auprès des ménages. Après avoir expliqué la définition des forêts secondaires adoptée dans le cadre de cette recherche, les questions ont été focalisées sur les diverses utilisations des bois issus de ces forêts secondaires. Un ménage est défini comme l'ensemble des différents membres, apparentés ou non, vivant ensemble dans le même logement, mettant en commun leurs ressources et satisfaisant en commun l'essentiel de leurs

Tableau I.

Les villages d'intervention.

\begin{tabular}{|c|c|c|c|}
\hline Village & $\begin{array}{l}\text { Localisation dans } \\
\text { le Menabe central }\end{array}$ & $\begin{array}{c}\text { Mode villageois } \\
\text { de gestion des forêts }\end{array}$ & Ethnie majoritaire \\
\hline Tsitakabasia & Nord & Forêt sous Gcf* & Sakalava (92 \%) \\
\hline Mandroatsy & Centre & Forêt sous Gcf* & Korao (100 \%) \\
\hline Anivorano & Sud & $\begin{array}{c}\text { Forêts secondaires } \\
\text { sans mode de gestion particulier }\end{array}$ & Antandroy (85 \%) \\
\hline
\end{tabular}




\section{Propriétés physico-mécaniques des bois de forêts secondaires}

besoins alimentaires et autres besoins vitaux (MAEP, 2003). Les ménages échantillons ont été choisis au hasard pour représenter un village. En moyenne, chaque village comporte 114 ménages. Pour répondre à la représentativité statistique, 40 ménages par village ont été interviewés, soit un taux d'échantillonnage de $35 \%$. Ensuite, des entretiens semi-directifs ont été adoptés pour les investigations sur les conditions d'accès aux ressources des forêts secondaires. Ces discussions ont été menées auprès de personnes-ressources, notamment les leaders, les notables dans les villages et les membres des associations gestionnaires des ressources naturelles. Des discussions informelles ont été également menées avec les villageois concernant leur perception des forêts secondaires en général. Ces éléments de discussion ont fourni des suppléments d'explication des faits énoncés pendant les enquêtes auprès des ménages.

Pour mesurer l'importance et la priorisation des essences par les villageois, la « méthode de distribution de cailloux » ou " scoring » inspirée de SHEIL et al. (2004) a été utilisée. Elle part du principe que les populations locales sont les meilleurs juges de ce qui est directement important pour elles, ce qui a été nécessaire au vu du nombre élevé d'espèces que les villageois prélèvent dans les forêts secondaires. Pour chaque catégorie d'utilisation du bois, chaque espèce obtient donc une note (en pourcentage du nombre total de mentions) qui correspond à son importance pour un ménage. Les notes données par chaque ménage pour chaque espèce ont ensuite été moyennées pour obtenir une valeur globale pour la région. La composition des groupes a été faite au hasard mais en considérant l'ethnie, le genre ainsi que l'âge.

Pour déterminer les espèces de forêts secondaires les plus importantes pour les villageois, toutes formes d'utilisation confondues, les critères de sélection du tableau II ont été appliqués sur la liste d'espèces évoquées par les villageois pour la satisfaction de leurs besoins quotidiens en bois. Les espèces les plus importantes doivent répondre à tous les seuils.
Les espèces de forêts secondaires qui ont été sélectionnées par l'application des critères du tableau II ont fait l'objet d'une caractérisation technologique selon les propriétés physiques et mécaniques du bois. La connaissance de ces propriétés, permettant d'estimer les potentialités de ces espèces, contribue à déterminer leur capacité de substitution technologique vis-à-vis des essences de la forêt primaire. Les essais ont porté sur les caractéristiques physiques (masse volumique à $12 \%$, retrait, dureté) et les caractéristiques mécaniques (module d'élasticité, contrainte de rupture en flexion, contrainte de rupture en compression). Les échantillons ont été préparés à partir de 5 arbres par espèce en ayant choisi les arbres de plus grands diamètres. Sur chacun de ces arbres, un billon de $80 \mathrm{~cm}$ a été prélevé à 1,30 m de hauteur. Chaque éprouvette a été prélevée du plateau diamétral en veillant à éviter la moelle et les défauts (fentes, nœuds, etc.), et de sorte à avoir les trois directions de référence du bois (longitudinale, radiale, tangentielle).

\section{Masse volumique}

La masse volumique a été évaluée en suivant la norme Afnor Nfb 51-005 (1985) sur des éprouvettes de 20 mm (R) x $20 \mathrm{~mm}(\mathrm{~T}) \times 20 \mathrm{~mm}(\mathrm{~L})$ à $12 \%$ d'humidité. Cette condition a été obtenue en stabilisant les éprouvettes dans une enceinte climatique réglée à une température de $20^{\circ} \mathrm{C}$ et à une humidité relative de $65 \%$ jusqu'à obtention d'une masse constante. La masse volumique est calculée par le rapport entre la masse obtenue à l'aide d'une balance d'une précision de 0,01 g et le volume mesuré avec un pied à coulisse d'une précision de 0,01 mm, selon la formule 1 .

$M V=\frac{M_{12}}{V_{12}}$

Avec :

$M V$ : masse volumique $\left(\mathrm{g} / \mathrm{cm}^{3}\right)$;

$M_{12}$ : masse de l'éprouvette à $12 \%$ d'humidité $(\mathrm{g})$;

$V_{12}$ : volume de l'éprouvette à $12 \%$ d'humidité $\left(\mathrm{cm}^{3}\right)$.

Tableau II.

Critères de détermination des espèces de forêts secondaires les plus utilisées par les villageois.

\begin{tabular}{l|l|l}
\hline Critères & Indicateurs & Seuil \\
\hline Valeur et utilité de la ressource & $\begin{array}{l}\text { Nombre de mentions dans } \\
\text { les interviews villageois }\end{array}$ & Au moins 12 mentions (10\%) \\
\hline $\begin{array}{l}\text { Importance de la ressource dans } \\
\text { la vie quotidienne des villageois }\end{array}$ & $\begin{array}{l}\text { Nombre de catégories d'utilisation, } \\
\text { tous terroirs confondus }\end{array}$ & Au moins 2 catégories d'utilisations \\
\hline Ordre de priorité villageois & $\begin{array}{l}\text { Dépouillement des exercices de « scoring » } \\
\text { dans les trois terroirs }\end{array}$ & $\begin{array}{l}\text { Parmi les } 10 \text { premiers produits les plus } \\
\text { importants selon les villageois }\end{array}$ \\
\hline Utilisations dans les terroirs & $\begin{array}{l}\text { Nombre de terroirs où la ressource } \\
\text { est utilisée }\end{array}$ & 3 terroirs \\
\hline Spécificité du lieu de collecte/service & $\begin{array}{l}\text { Part de l'extraction de la ressource } \\
\text { dans les forêts secondaires }\end{array}$ & $\begin{array}{l}\text { Au moins } 75 \% \text { de l'extraction de la ressource } \\
\text { se fait dans les forêts secondaires }\end{array}$
\end{tabular}




\section{Mesure des retraits}

La variation de longueur dans les directions d'anisotropie a été quantifiée par le retrait tangentiel Rt et le retrait radial $R r$. L'essai a été réalisé sur des éprouvettes de $20 \mathrm{~mm}$ (R) $\times 20 \mathrm{~mm}(\mathrm{~T}) \times 50 \mathrm{~mm}(\mathrm{~L})$ en suivant la norme Afnor Nfb 51-006 (1985). Les dimensions et masses des éprouvettes ont été relevées à l'état saturé puis à l'état anhydre (dans une étuve ventilée à $103^{\circ} \mathrm{C}$ jusqu'à obtention d'une masse constante). Les retraits totaux ont ensuite été calculés selon la formule 2 .

$R(\%)=\frac{D_{s}-D_{o}}{D_{s}} \times 100$

avec :

$D$ s et $D o$ : respectivement les dimensions des échantillons dans la direction considérée à l'état saturé puis à l'état anhydre $(\mathrm{mm})$.

\section{Mesure du module d'élasticité}

Le module d'élasticité a été obtenu à l'aide d'une méthode non destructive mettant en œuvre le dispositif Bing développé au Cirad (BORDONNÉ, 1989). Ce dispositif permet de mesurer le module d'élasticité d'une éprouvette sans défaut, mesurant $20 \mathrm{~mm}(\mathrm{R}) \times 20 \mathrm{~mm}(\mathrm{~T}) \times 360 \mathrm{~mm}(\mathrm{~L})$ et à $12 \%$ d'humidité, par analyse du spectre de fréquence de résonance.

\section{Mesure des contraintes de rupture en flexion et en compression}

Les mêmes éprouvettes, ayant servi à mesurer le module d'élasticité, ont été utilisées pour mesurer la contrainte de rupture en flexion statique 4 points $F_{12}$ selon la formule 3. Le protocole d'essai était conforme à la norme Afnor Nfb 51-008 (1987). La charge a été appliquée sur la face LT des éprouvettes à $12 \%$ d'humidité.

$F_{12}=\frac{3 F_{\max }(l-a)}{2 b h^{2}}$

avec :

$F_{12}$ : contrainte de rupture en flexion statique 4 points (MPa) ;

$F_{\text {max }}:$ charge totale appliquée à la rupture en flexion $(\mathrm{N})$;

$l$ : distance entre les axes des appuis cylindriques $(\mathrm{mm})$;

$a$ : distance entre les axes des têtes de chargement $(\mathrm{mm})$;

$b$ : largeur de l'éprouvette $(\mathrm{mm})$;

$h$ : hauteur de l'éprouvette $(\mathrm{mm})$.

Des éprouvettes de $20 \mathrm{~mm}(\mathrm{R})$ x $20 \mathrm{~mm}(\mathrm{~T}) \times 100 \mathrm{~mm}$ (L) ont ensuite été prélevées sur les éprouvettes de flexion afin de déterminer la contrainte de rupture en compression $C_{12}$ en suivant la formule 4 , selon la norme Afnor $\mathrm{Nfb} 51$ 007 (1985).

$C_{12}=\frac{P}{b h}$ avec :

$C_{12}$ : contrainte de rupture en compression $(\mathrm{MPa})$;

$P$ : charge maximale $(\mathrm{N})$;

$b$ : largeur de l'éprouvette $(\mathrm{mm})$;

$h$ : hauteur de l'éprouvette $(\mathrm{mm})$.

\section{Mesure de la dureté Monnin}

La dureté Monnin $D$ a été mesurée en suivant la norme Afnor Nfb 51-013 (1985), selon la formule 5. La largeur de l'empreinte a été mesurée par analyse d’image.

$D=\frac{1}{15-\frac{1}{2} \sqrt{900-c^{2}}}$

avec :

$D$ : dureté ;

$c$ : largeur de l'empreinte $(\mathrm{mm})$.

Les propriétés ainsi mesurées à partir des bois de forêts secondaires ont fait l'objet d'une étude comparative d'analyse en composantes principales par rapport aux données disponibles pour quatre espèces de forêts primaires menacées de disparition : Hernandia voyroni, Dalbergia spp., Cedrelopsis grevei et Commiphora mafaiboa. Le choix de ces quatre espèces repose sur les données de la littérature, les résultats des discussions informelles et les observations directes sur le marché du bois de Morondava qui est le chef-lieu de la région. Avant 1988, Hernandia voyroni était l'espèce la plus appréciée. Elle était surtout utilisée pour les éléments constructifs structuraux des maisons (piliers principaux), les portes et fenêtres ainsi que les cercueils. Depuis 1988, cette espèce n'est plus apparue sur le marché du bois de Morondava et a été remplacée par d'autres essences, notamment Dalbergia spp. et Commiphora mafaiboa, qui fournissent la plus grande partie du bois utilisé dans la région (RANDRIANASOLO et al., 1996). La promotion de l'espèce Commiphora mafaiboa sur le marché du bois dans le Menabe a été du ressort du Centre de formation professionnelle forestière de Morondava ${ }^{1}$, qui a mené des recherches en technologie du bois sur différentes espèces de forêts primaires peu connues des consommateurs afin de diminuer les pressions sur les espèces trop exploitées (ANDRIAMBELO, 2005).

${ }^{1}$ Aujourd'hui Cnferef ou Centre national de formation, d'étude et de recherche en environnement et foresterie.

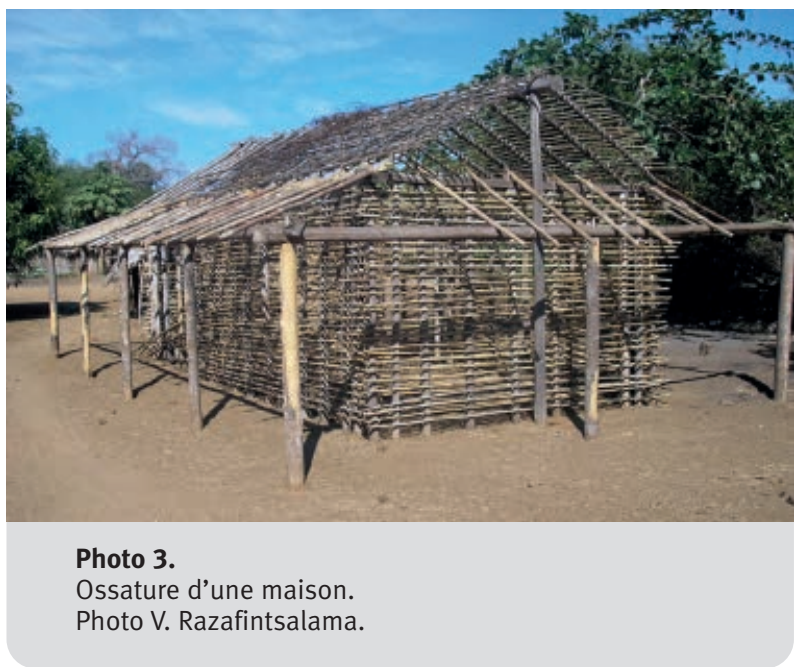




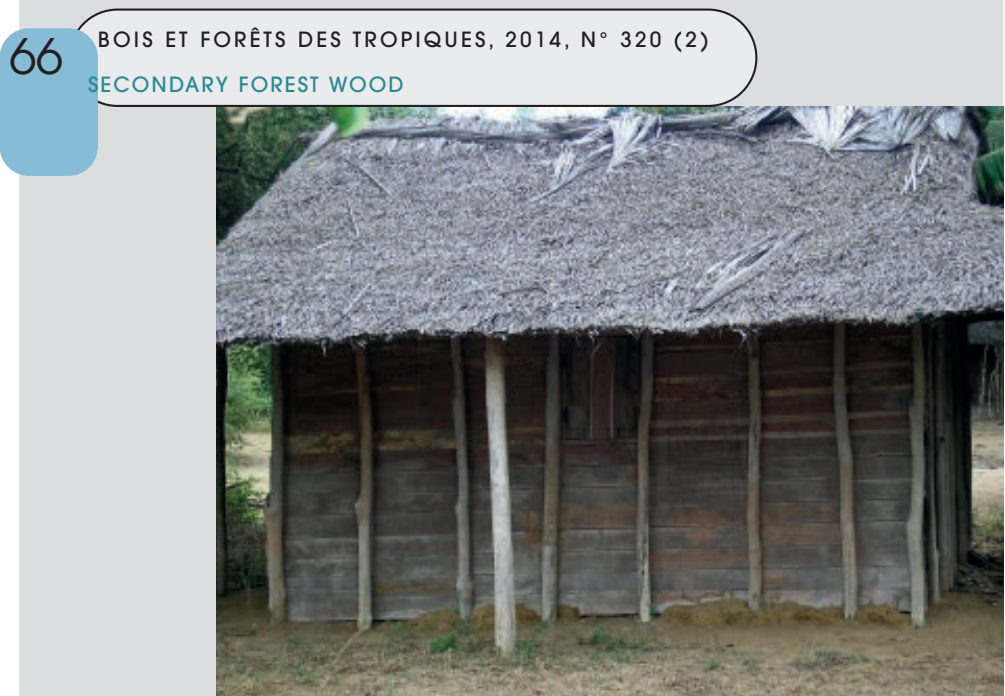

Photo 4.

Maison avec des murs en bois.

Photo V. Razafintsalama.

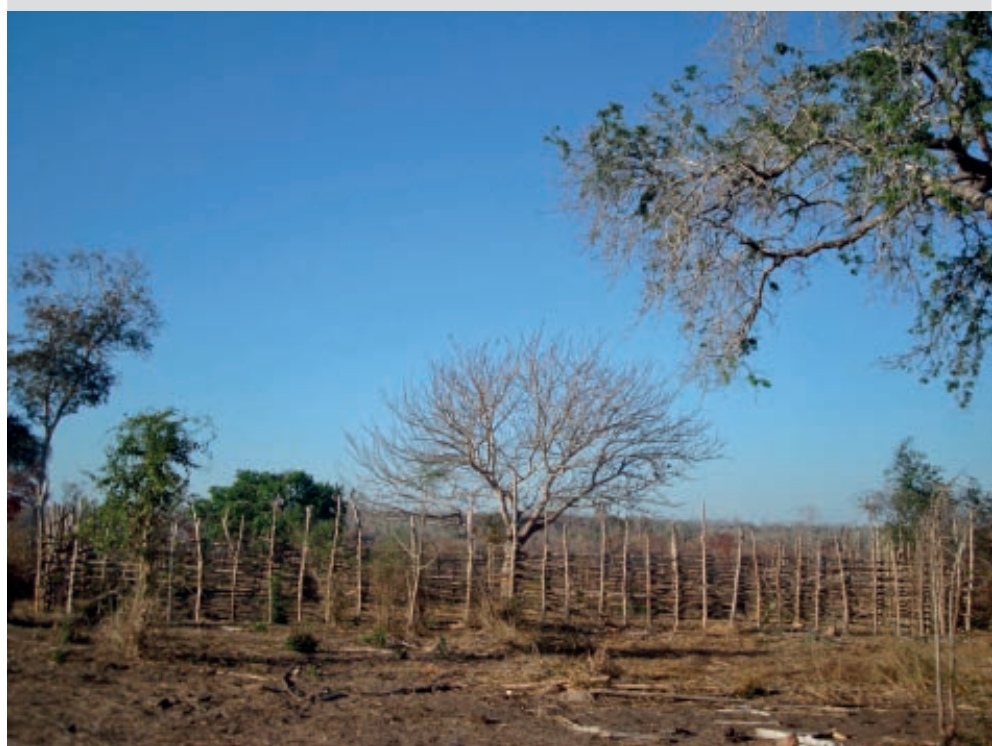

Photo 5.

Parc à bœufs.

Photo V. Razafintsalama.

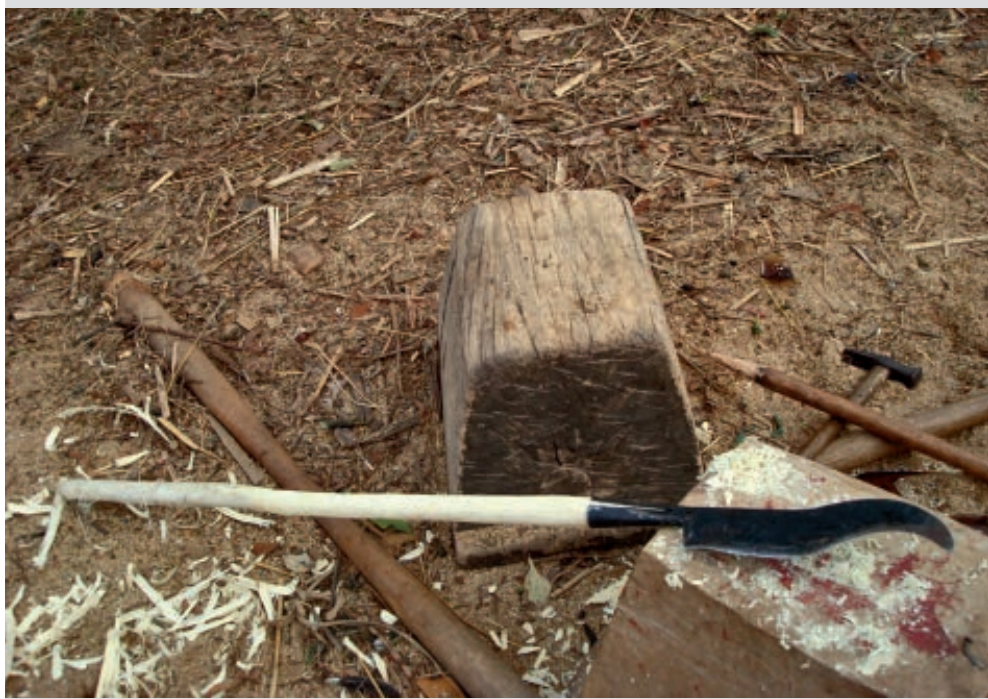

Photo 6.

Utilisation de Rhopalocarpus lucidus pour renouveler un manche d'outil.

Photo V. Razafintsalama.

\section{Résultats}

\section{Les catégories d'utilisation des bois de forêts secondaires}

Les enquêtes ont révélé que les bois prélevés dans les forêts secondaires sont utilisés suivant trois catégories : les constructions permanentes, les constructions légères et les sources d'énergie.

\section{Constructions permanentes}

Le bois de construction constitue l'un des principaux produits forestiers ligneux recherchés par les populations rurales. C'est un élément indispensable pour la construction de maisons dans les villages du Menabe central (photo 3). Les piliers principaux et secondaires, les traverses et les charpentes du toit sont constitués par des perches de dimensions diverses variant de $5 \mathrm{~cm}$ pour les traverses à environ $15 \mathrm{~cm}$ pour les piliers principaux (photo 4).

\section{Constructions légères}

Les cases temporaires dans les champs de culture, les clôtures des champs, les parcs à bœufs (photo 5) et les manches d'outils (photo 6) sont groupés dans la catégorie des constructions légères. Les cases temporaires sont conçues pour être provisoires, généralement pour une campagne de culture. Mais pratiquement, les paysans y passent la majorité de leur temps durant l'année, pendant les différents travaux agricoles. Ces maisons peuvent même être déplacées. Les clôtures des champs sont construites notamment pour éviter la divagation des animaux (potamochères, bovins ou caprins non gardés) dans les cultures. Tous les manches d'outils (bêches, haches, coupe-coupe) sont fabriqués en bois par l'utilisateur. Les diamètres nécessaires étant entre 3 et $4 \mathrm{~cm}$, des branches suffisent parfois à fabriquer ces manches d'outils.

\section{Bois d'énergie}

Il s'agit du bois de chauffe (photos 7 et 8 ) et du charbon de bois (photo 9). Les ménages ruraux collectent euxmêmes leur bois de chauffe qui est destiné entièrement à leur propre consommation. Aucune réglementation n'est appliquée concernant la collecte de bois de chauffe étant donné qu'il s'agit de bois mort. La population urbaine couvre ses besoins énergétiques en utilisant principalement le charbon de bois, compte tenu de sa compétitivité élevée au regard des autres types d'énergie comme le gaz. La production est totalement destinée à la vente dans le but de satisfaire les besoins monétaires immédiats.

\section{Les principales essences utilisées}

Pour les utilisations en construction, les enquêtes ont révélé quatre critères pour le choix des espèces, les propriétés mécaniques du bois, la résistance du bois aux champignons et insectes xylophages (durabilité naturelle), la rectitude du fût et la disponibilité de l'espèce dans les lieux de prélèvement. L'application de ces critères permettrait aux propriétaires de disposer potentiellement d'une maison 


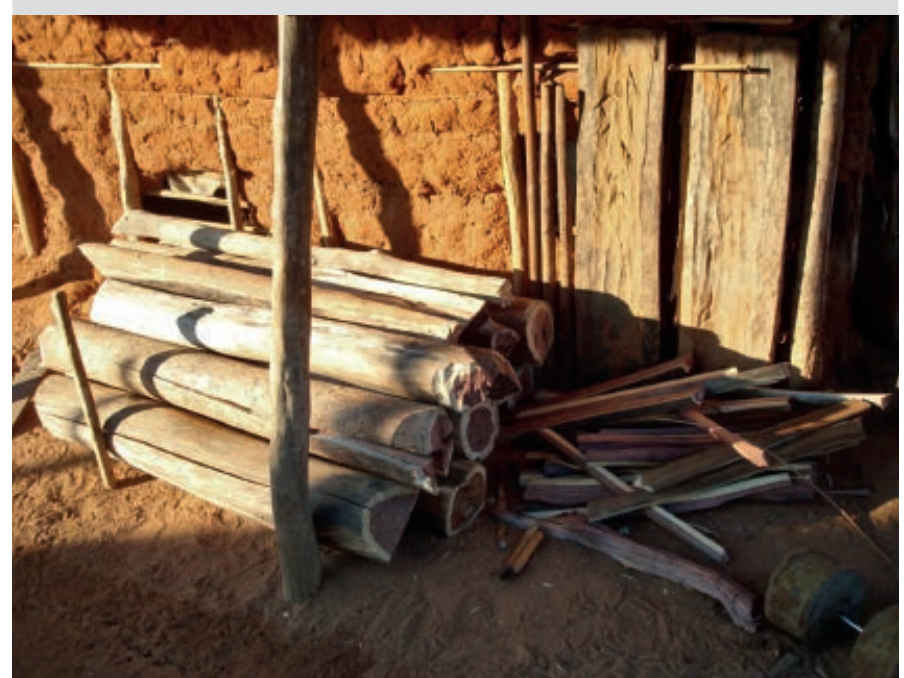

Photo 7.

Bois précieux (Dalbergia sp.) utilisé comme bois de chauffe. Photo V. Razafintsalama.

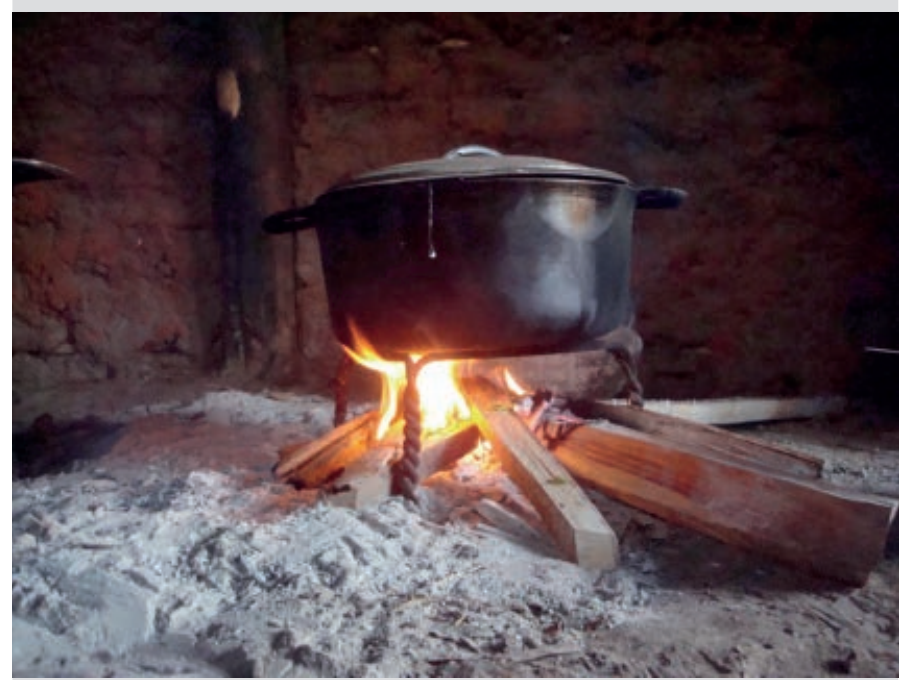

Photo 8.

Utilisation des bois de chauffe.

Photo V. Razafintsalama.

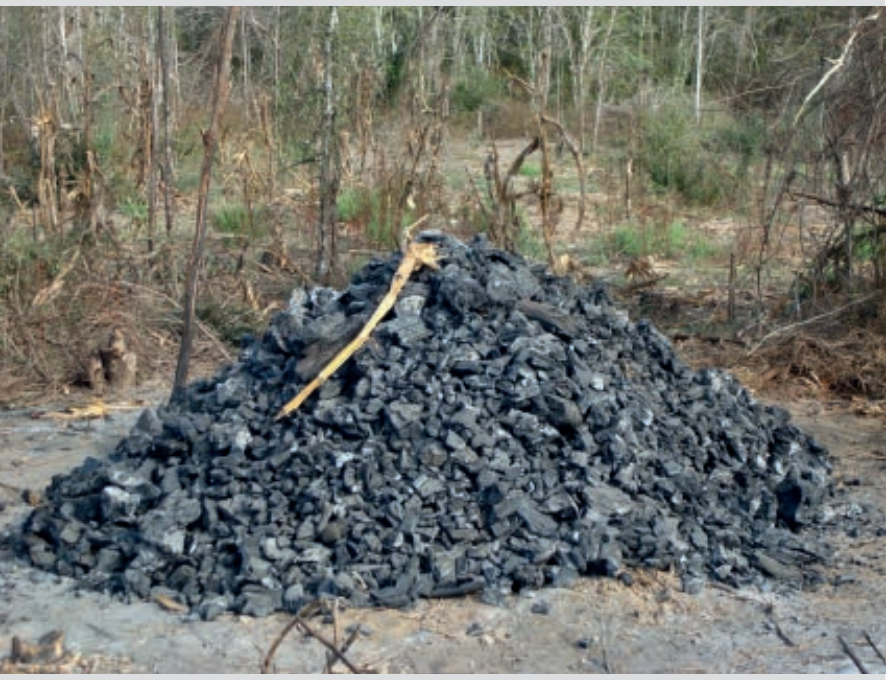

Photo 9.

Meule de charbon de bois en cours de déchargement dans les forêts secondaires.

Photo V. Razafintsalama. durable. Le tableau III et la figure 2 représentent les espèces prélevées dans les forêts secondaires, et qui sont les plus utilisées pour chaque catégorie d'utilisation. On remarque que, parmi les espèces citées, il en existe qui sont plutôt des espèces de forêt primaire (" prim./sec. ») et d'autres qui sont fréquentes en forêt secondaire mais qui peuvent être rencontrées en forêt primaire également ("sec./prim. »). Pour le choix d'espèces de substitution potentielles, l'étude s'est focalisée sur des espèces exclusivement de forêt secondaire (" secondaire »).

Malgré l'utilisation des bois de forêt secondaire, la préférence en espèces est dominée par les espèces de la forêt primaire et est toujours corollaire d'une forte exploitation entraînant leur raréfaction.

Le tableau III et la figure 2 a montrent que les espèces des forêts secondaires ne sont pas très renommées en construction. L'espèce Cedrelopsis grevei (CG) est la plus utilisée aussi bien pour la charpenterie que pour les piliers. Elle se trouve avec deux autres espèces, Securinega seyrigii (SS) et Cedrelopsis gracilis (CGR), dans la catégorie des espèces les plus utilisées. L'espèce de forêt secondaire Grewia picta (GP) a été évoquée mais cette espèce n'est pas la plus désirée par les villageois.

Les constructions légères ne sont pas très exigeantes en termes d'espèces, notamment pour les clôtures des champs, mais des préférences sont évoquées par les villageois. Le tableau III et les figures $2 c$ et $2 d$ font ressortir que, pour toutes les constructions légères, deux espèces sont très utilisées : Ziziphus mauritiana, ZM (forêt secondaire), et Capuronia madagascariensis, CaM (forêt primaire/secondaire). La première espèce est vouée en grande partie à la construction de cases temporaires dans les champs à raison de $42,5 \%$ tandis que la seconde est plus utilisée pour les parcs à bœufs ( $40 \%)$. Concernant les clôtures des champs de culture (figure $2 \mathrm{~d}$ ), bien que cette forme de construction soit moins exigeante en caractéristiques mécaniques, l'utilisation abondante de Ziziphus mauritiana (ZM) s'explique par le fait que cette espèce porte des épines et que, une fois entassées, les branches constituent des ceintures épaisses permettant d'éviter la divagation des animaux. Concernant les manches d'outils (figure 2c), l'espèce de forêt secondaire Rhopalocarpus lucidus (RL) est largement plus appréciée du fait qu'elle est la plus facile à trouver (photo 6). En effet, l'arbre de cette espèce rejette de souche et les faibles dimensions nécessaires pour les manches d'outils sont commodément satisfaites. Grewia cyclea (GC) rejette aussi de souche mais son bois est plus tendre et moins longévif.

Pour le bois d'énergie (figure $2 b$ ), les enquêtes ont mis en évidence le fait que tous les bois morts provenant aussi bien de forêt primaire que secondaire peuvent être ramassés et utilisés comme bois de chauffe et que tous les arbres ayant atteint $5 \mathrm{~cm}$ de diamètre sont utilisés pour la carbonisation. Cependant, des prédilections sont indiquées par les villageois (tableau III).

Pour le bois de chauffe (figure $2 \mathrm{~b}$ ), les villageois préfèrent les bois durs qui se consument lentement et qui brûlent bien, même en période de pluie. C'est le cas des espèces de forêt primaire Cedrelopsis grevei (CG) et Dalbergia spp. (DS) (photo 7 ). D'après la figure $2 b$, seule l'espèce Ziziphus 
Tableau III.

Espèces les plus utilisées pour chaque catégorie d’utilisation, qui sont collectées dans les forêts secondaires.

\begin{tabular}{|c|c|c|c|c|c|}
\hline \multirow[t]{2}{*}{ Ouvrage } & \multicolumn{3}{|c|}{ Espèces } & \multirow{2}{*}{$\begin{array}{l}\text { Pourcentage d’utilisation } \\
\text { par les ménages }\end{array}$} & \multirow{2}{*}{$\begin{array}{l}\text { Type de forêt } \\
\text { de collecte* }\end{array}$} \\
\hline & Noms vernaculaires & Noms scientifiques & Initiale & & \\
\hline \multirow[t]{8}{*}{ Charpente } & Katrafay & Cedrelopsis grevei Baill. & CG & 33 & Prim./sec. \\
\hline & Anatsioky & Securinega seyrigii Leandri & SS & 13 & Prim./sec. \\
\hline & Mampandry & Cedrelopsis gracilis J.-F. Leroy & CGR & 10 & Prim./sec. \\
\hline & Amaninomby & Terminalia fatraea (Poir.) DC. & TF & 5 & Sec./prim. \\
\hline & Taly & Terminalia tricristata Capuron & TC & 3 & Prim./sec. \\
\hline & Kitata & Capuronia madagascariensis Lourteig & CAM & 2 & Prim./sec. \\
\hline & Hazomby & Strychnos decussata (Pappe) Gilg & SD & 2 & Sec./prim. \\
\hline & Hazomena & Securinega perrieri Leandri & SP & 2 & Prim./sec. \\
\hline \multirow[t]{6}{*}{ Piliers } & Katrafay & Cedrelopsis grevei Baill. & CG & 45 & Prim./sec. \\
\hline & Manary & Dalbergia spp. & DS & 4 & Prim./sec. \\
\hline & Hazomena & Securinega perrieri Leandri & $\mathrm{SP}$ & 3 & Prim./sec. \\
\hline & Kitata & Capuronia madagascariensis Lourteig & CAM & 3 & Prim./sec. \\
\hline & Tsilaitsy & Noronhia alleizettei Dubard & NA & 2 & Prim./sec. \\
\hline & Sely & Grewia picta Baill. & GP & 2 & Secondaire \\
\hline \multirow{5}{*}{$\begin{array}{l}\text { Cases } \\
\text { temporaires }\end{array}$} & Konazy & Ziziphus mauritiana Lam. & ZM & 43 & Secondaire \\
\hline & Kitata & Capuronia madagascariensis Lourteig & CAM & 18 & Prim./sec. \\
\hline & Mampandry & Cedrelopsis gracilis J.-F. Leroy & CGR & 8 & Prim./sec. \\
\hline & Katrafay & Cedrelopsis grevei Baill. & CG & 5 & Prim./sec. \\
\hline & Namoloagna & Foetida retusa Blume & FR & 5 & Secondaire \\
\hline Clôtures & Konazy & Ziziphus mauritiana Lam. & ZM & 30 & Secondaire \\
\hline & Manary & Dalbergia spp. & DS & 8 & Prim./sec. \\
\hline & Konazy & Ziziphus mauritiana Lam. & ZM & 8 & Secondaire \\
\hline \multirow{5}{*}{$\begin{array}{l}\text { Manches } \\
\text { d'outils }\end{array}$} & Talafotsy & Rhopalocarpus lucidus Bojer & $\mathrm{RL}$ & 44 & Secondaire \\
\hline & Katrafay & Cedrelopsis grevei Baill. & CG & 15 & Prim./sec. \\
\hline & Latabarika & Grewia cyclea Baill. & GC & 14 & Secondaire \\
\hline & Manary & Dalbergia spp. & DS & 7 & Prim./sec. \\
\hline & Amaninomby & Terminalia fatraea (Poir.) DC. & TF & 4 & Sec./prim. \\
\hline \multirow{5}{*}{$\begin{array}{l}\text { Bois } \\
\text { de chauffe }\end{array}$} & Katrafay & Cedrelopsis grevei Baill. & CG & 53 & Prim./sec. \\
\hline & Manary & Dalbergia spp. & DS & 42 & Prim./sec. \\
\hline & Konazy & Ziziphus mauritiana Lam. & ZM & 39 & Secondaire \\
\hline & Anatsioky & Securinega seyrigii Leandri & SS & 12 & Prim./sec. \\
\hline & Sely & Grewia picta Baill. & GP & 7 & Secondaire \\
\hline \multirow{7}{*}{$\begin{array}{l}\text { Charbon } \\
\text { de bois }\end{array}$} & Kily & Tamarindus indica $\mathrm{L}$. & $\mathrm{Tl}$ & 68 & Sec./prim. \\
\hline & Konazy & Ziziphus mauritiana Lam. & ZM & 54 & Secondaire \\
\hline & Hazomena & Securinega perrieri Leandri & SP & 40 & Prim./sec. \\
\hline & Talafotsy & Rhopalocarpus lucidus Bojer & $\mathrm{RL}$ & 36 & Secondaire \\
\hline & Kininina & Eucalyptus spp. & ES & 33 & Reboisement \\
\hline & Kitata & Capuronia madagascariensis Lourteig & $\mathrm{CM}$ & 18 & Prim./sec. \\
\hline & Taly & Terminalia tricristata Capuron & TC & 11 & Prim./sec. \\
\hline
\end{tabular}

* Le type de forêt cité en premier désigne la forêt de collecte la plus fréquente (prim. : primaire ; sec. : secondaire). 


\section{Choix et propriétés des essences de forêt secondaire}

mauritiana (ZM) est utilisée pour les deux formes de bois d'énergie (bois de chauffe et charbon de bois). Elle est ainsi classée parmi les espèces les plus appréciées pour le charbon de bois (photo 9), avec l'espèce de forêt secondaire Rhopalocarpus lucidus (RL). Elle figure avec Dalbergia spp. (DS) et Cedrelopsis grevei (CG) dans la catégorie des espèces qui sont les plus utilisées par la population pour un usage de bois de chauffe. Les deux autres espèces de forêt secondaire, Terminalia tricristata (TC) pour le charbon de bois et Grewia picta (GP) pour le bois de chauffe, méritent d'être mieux connues et promues étant donné qu'elles sont aussi utilisées par la population, même en second choix.

Pour récapituler, les différentes observations permettent de constater que les villageois appliquent une priorisation de leurs choix, tributaire de certains critères basés sur leurs connaissances empiriques et sur la disponibilité des espèces. Les bois de forêt secondaire contribuent largement à satisfaire les besoins actuels en bois de la population locale. Ces résultats renforcent ainsi les observations de DE LAS SALAS (2002) concernant l'importance et le potentiel des forêts secondaires.
L'application des critères du tableau II a permis d'identifier les espèces de forêt secondaire les plus importantes pour les villageois du Menabe central. Trois espèces ont répondu aux conditions: Grewia picta (GP), Rhopalocarpus lucidus (RL) et Ziziphus mauritiana (ZM) (photos 10 et 11). Leurs propriétés n'ont jamais été caractérisées auparavant (GUENEAU, GUENEAU, 1969 ; GRISA, 1988 ; RAKOTOVAO et al., 2012). C'est ce qui a motivé les essais exploratoires menés dans cette étude. Les résultats des caractérisations physiques et mécaniques des bois de ces essences sont présentés dans le tableau IV.

Les masses volumiques des trois espèces de forêts secondaires sont de $0,56 \mathrm{~g} / \mathrm{cm}^{3}$ pour Grewia picta, $0,67 \mathrm{~g} / \mathrm{cm}^{3}$ pour Ziziphus mauritiana et $0,87 \mathrm{~g} / \mathrm{cm}^{3}$ pour Rhopalocarpus lucidus. Grewia picta est donc un bois mi-dense tandis que Ziziphus mauritiana et Rhopalocarpus lucidus sont des bois denses. Grewia picta et Ziziphus mauritiana sont des bois midurs et Rhopalocarpus lucidus est un bois dur. Les retraits radiaux sont moyens (4,1 à 5,5\%). Le coefficient d'anisotropie

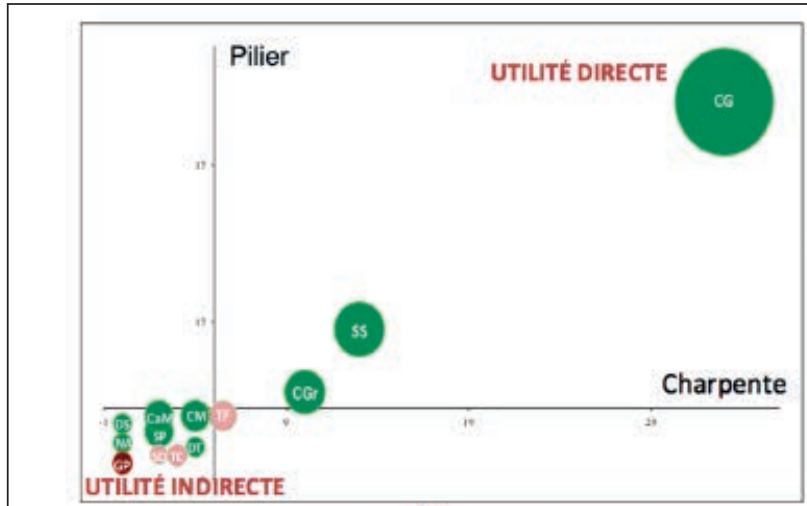

(a)

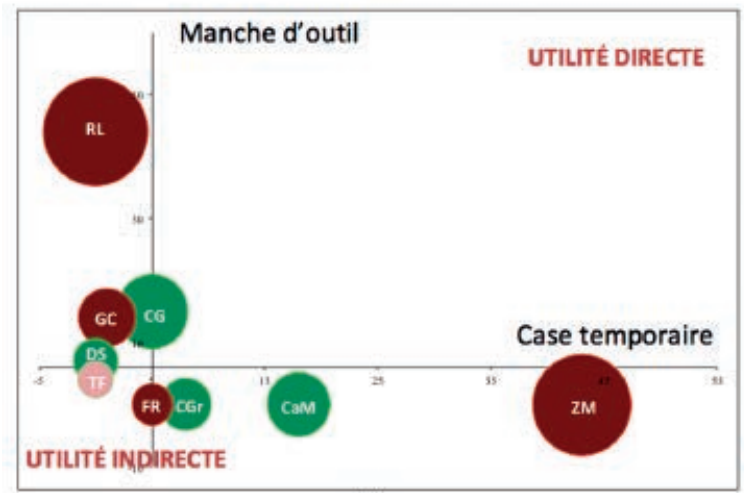

(c)

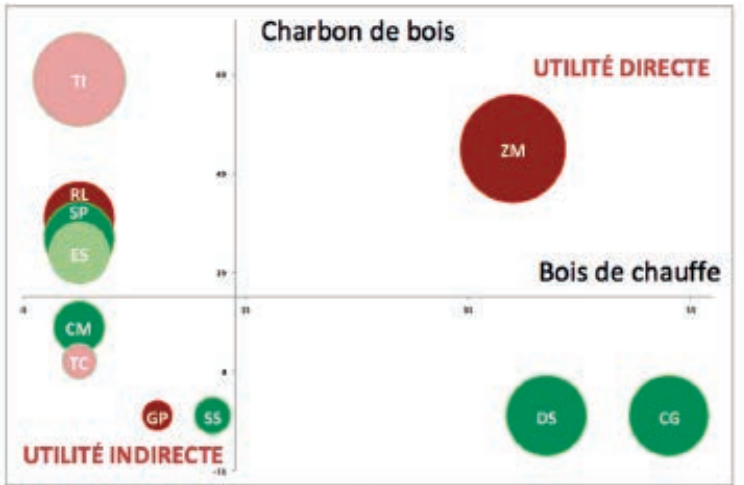

(b)

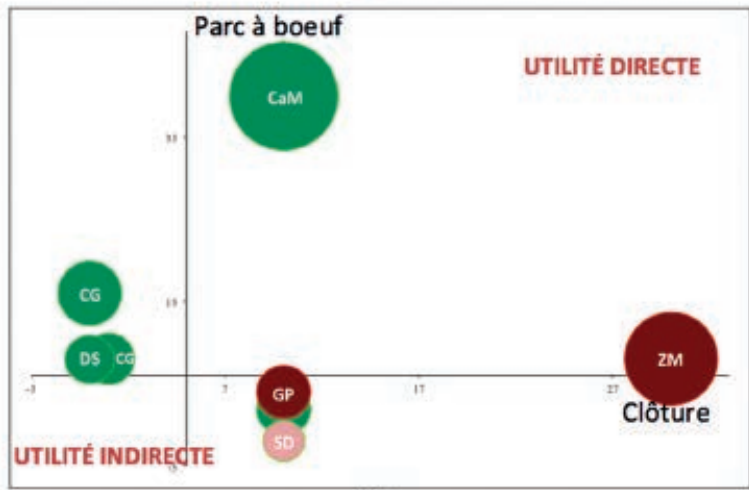

(d)

Primaire/Secondaire

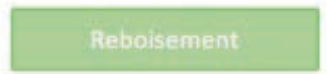

Figure 2.

Utilités des espèces (a) pour les constructions permanentes, (b) pour le bois d'énergie, (c) (d) pour les constructions légères. Les légendes utilisées dans la figure sont les initiales des noms scientifiques des espèces (cf. tableau III).

Les axes des abscisses et des ordonnées représentent les pourcentages d'utilisation de chaque espèce mentionnés par les villageois selon chaque catégorie d'utilisations. Le diamètre des cercles représente une pondération de ces pourcentages selon les deux catégories d'utilisations. 


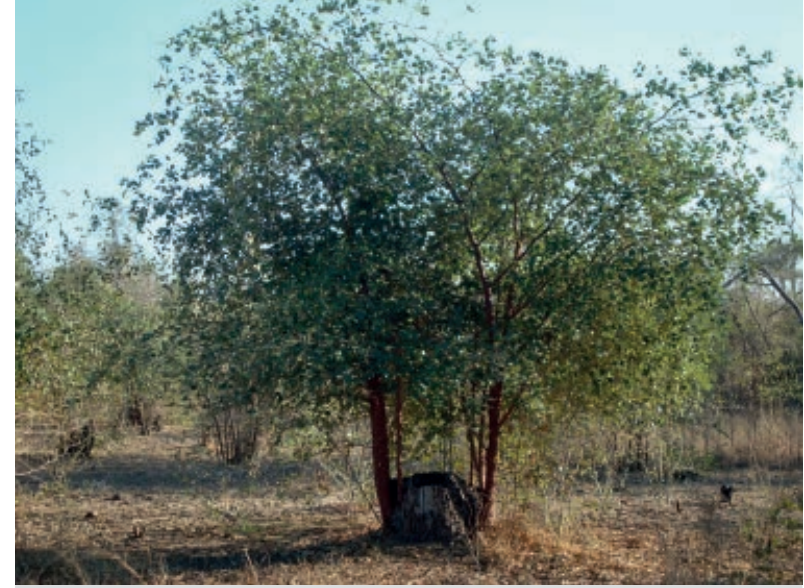

Photo 10.

Rejets de souche de Ziziphus mauritiana.

Photo V. Razafintsalama.

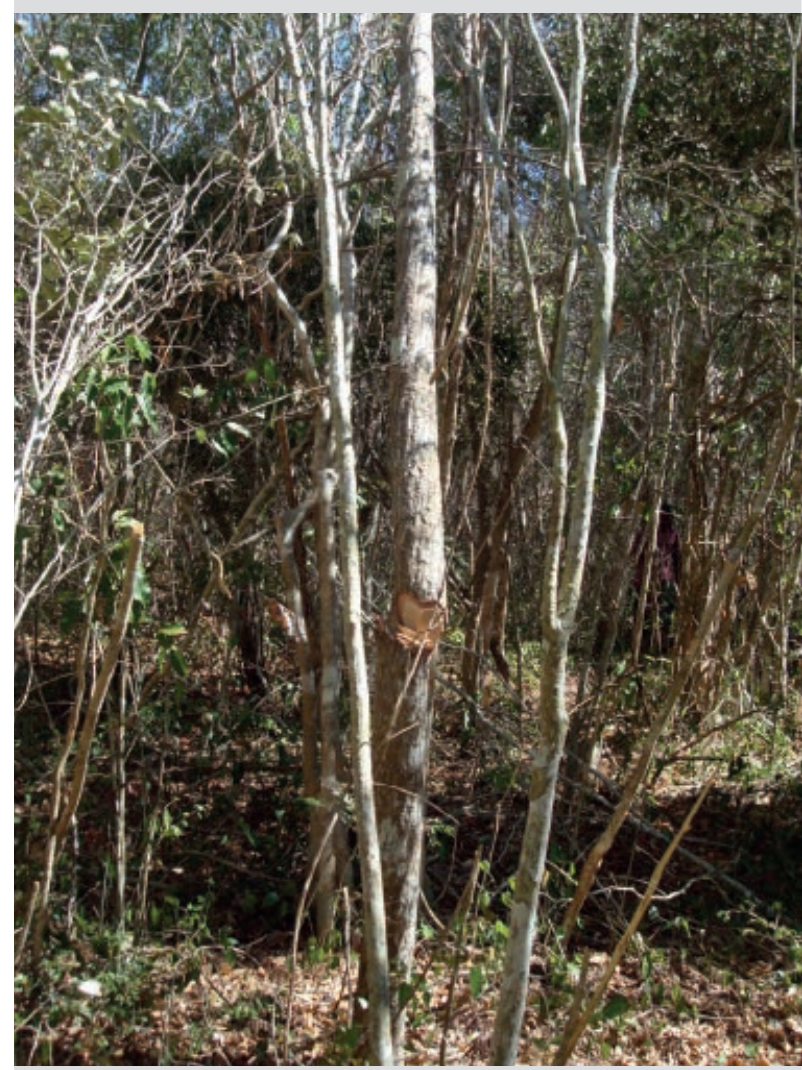

Photo 11.

Pieds de Grewia picta.

Photo V. Razafintsalama.

est faible. Cette faible anisotropie du retrait peut s'expliquer par le fait que les éprouvettes ont été prélevées près du cœur en raison du faible diamètre des arbres. Elle n'est peutêtre pas reproductible pour des arbres de plus gros diamètre. Concernant les propriétés mécaniques, les trois essences de forêt secondaire présentent des modules d'élasticité moyens (de $10732 \mathrm{MPa}$ à $13914 \mathrm{MPa}$ ), des contraintes de rupture en compression moyennes (de 46,8 MPa à $50,1 \mathrm{MPa}$ ) et des contraintes de rupture en flexion statique moyennes (de 95,1 MPa à 130,3 MPa).

\section{Discussion}

Au vu de ces résultats, il est possible d'avancer que les trois essences de forêts secondaires les plus importantes dans les utilisations villageoises actuelles possèdent des propriétés physico-mécaniques moyennes à élevées, ce qui permettrait une valorisation de ces espèces pour différentes utilisations. Leurs caractéristiques physiques et mécaniques ont été comparées à celles des données de la littérature concernant les essences des forêts primaires qui se raréfient ou qui sont protégées, et qui sont pourtant les plus convoitées pour divers usages dans la région du Menabe (tableau IV et figure 3). Il est à noter que les tests physicomécaniques ont été effectués à partir d'arbres de faible diamètre (inférieur à $21 \mathrm{~cm}$ ) alors que les données sur les bois de forêts primaires ont été obtenues sur de gros arbres ayant des diamètres supérieurs à $40 \mathrm{~cm}$. II serait possible de compter sur des propriétés supérieures pour les bois de forêts secondaires au cas où il se serait agi d'arbres de diamètre équivalent (supérieur à $40 \mathrm{~cm}$ ). Quoi qu'il en soit, l'échantillon étudié représente le diamètre et l'âge des arbres faisant l'objet de l'exploitation courante et les données sont ainsi représentatives des propriétés des ressources sur le terrain.

L'analyse en composantes principales (figure 3) montre que la première composante principale contient $81 \%$ de la variance totale et la seconde composante principale $14 \%$. Puisque la représentation sur ces deux composantes concerne $95 \%$ de l'information totale, il suffit donc d'étudier les deux. Sur le cercle de corrélation (figure 3a), toutes les variables, exceptée $R r$, sont anticorrélées à la première composante principale. La projection des individus dans le plan défini par ces deux axes (figure $3 \mathrm{~b}$ ) indique que l'axe 1 oppose les bois de forêt primaire très denses (Cedrelopsis grevei et Dalbergia spp.) aux bois de forêts primaires peu denses (Commiphora sp. et Hernandia voyroni). Les espèces de forêts secondaires étudiées se situent entre les deux catégories, avec l'espèce Rhopalocarpus lucidus qui est plus proche de Cedrelopsis grevei et Dalbergia spp. La masse volumique étant corrélée, plus ou moins étroitement, avec la dureté, le module d'élasticité, la contrainte de rupture en flexion et la contrainte de rupture en compression, cette tendance se retrouve sur ces autres paramètres. En effet, par exemple, les bois de forêt secondaire présentent un module d'élasticité considéré comme "moyen ", les situant entre les deux bois de forêt primaire qui ont un module d'élasticité faible (Commiphora sp. et Hernandia voyroni) et les deux bois de forêt primaire qui ont un module d'élasticité fort (Cedrelopsis grevei et Dalbergia spp.). Le paramètre $\mathrm{Rr}$ est anticorrélé à la seconde composante principale. L'axe 2 met en évidence l'opposition qui existe entre les quatre essences de forêt primaire et les trois essences de forêt secondaire. Le retrait radial est beaucoup plus fort sur ces dernières. L'explication peut être le faible diamètre et la proximité de la moelle pour les espèces de forêt secondaire comme évoqué précédemment. Il est donc possible de s'apercevoir que les résultats des tests physico-mécaniques sur les bois de forêt secondaire les plus cités par 
les villageois sont conformes aux utilisations actuelles. Ces résultats permettent d'avancer que ces trois espèces de forêt secondaire figurent parmi les espèces de substitution potentielles. Des analyses complémentaires, notamment de la durabilité naturelle du bois, permettront de mieux préciser les potentialités de ces espèces de forêts secondaires à substituer efficacement à certaines espèces de forêt primaire dans le Menabe central. En outre, il serait intéressant de poursuivre cette étude en augmentant le nombre d'échantillons et d'arbres considérés de façon à mieux prendre en compte les variabilités inter-arbres.

Selon FINEGAN (1992), dans le cas du Costa Rica, les bois de forêt secondaire appartenant à la première phase de succession sont relativement légers et de qualité médiocre par rapport aux bois de forêt primaire. De même, EDWIN (2011) a trouvé que les bois de forêt secondaire en Papouasie-Nouvelle-Guinée possèdent des propriétés mécaniques (module d'élasticité et module de rupture) plus faibles que les bois de forêt primaire. Dans cette étude, le fait d'avoir choisi les essences à étudier en tenant compte des connaissances empiriques de la population a permis de déceler des essences avec de bonnes propriétés, équivalentes à celles des bois de forêts primaires. Ces résultats sont conformes aux résultats obtenus par PAREDES (2011), selon lesquels quatre espèces peu connues prélevées sélectivement dans les forêts secondaires en Colombie sont équivalentes aux bois de forêt primaire en termes de propriétés physiques (infradensité) et mécaniques (contraintes de rupture en flexion et en compression).

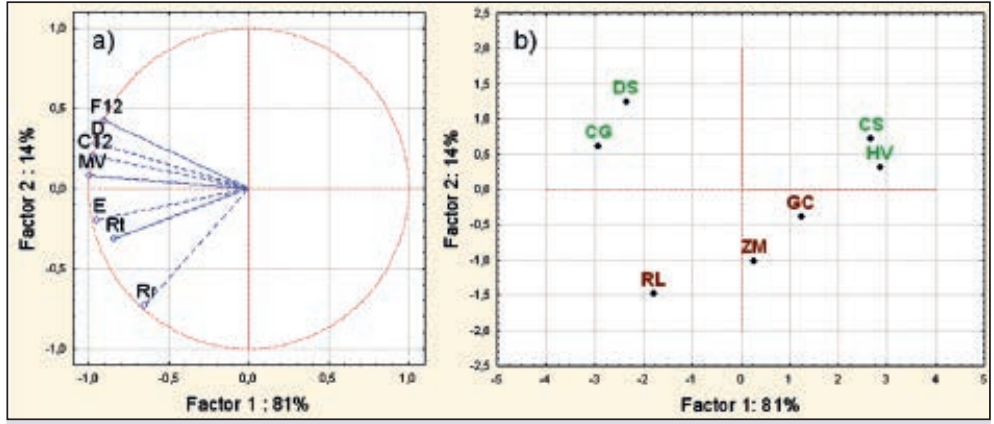

Figure 3.

Analyse en composantes principales - projection sur le plan factoriel principal : a) des variables, b) des individus.

En grenat : espèces de forêts secondaires ;

en vert : espèces de forêts primaires/secondaires.

Abréviations des espèces :

GP : Grewia picta ;

$\mathrm{RL}$ : Rhopalocarpus lucidus ;

ZM : Ziziphus mauritiana ;

CS : Commiphora sp. ;

$\mathrm{HV}$ : Hernandia voyroni;

CG : Cedrelopsis grevei ;

DS : Dalbergia spp.

Abréviations des propriétés :

MV : masse volumique à $12 \%$;

Rr : retrait radial total ;

Rt : retrait tangentiel total ;

D : dureté Monnin ; E : module d'élasticité ;

$\mathrm{C}_{12}$ : contrainte de rupture en compression axiale ;

$\mathrm{F}_{12}$ : contrainte de rupture en flexion statique 4 points.

\section{Tableau IV.}

Comparaison des caractéristiques du bois des trois essences de forêt secondaire les plus importantes pour les villageois avec les données de la littérature concernant des essences de référence des forêt primaire.

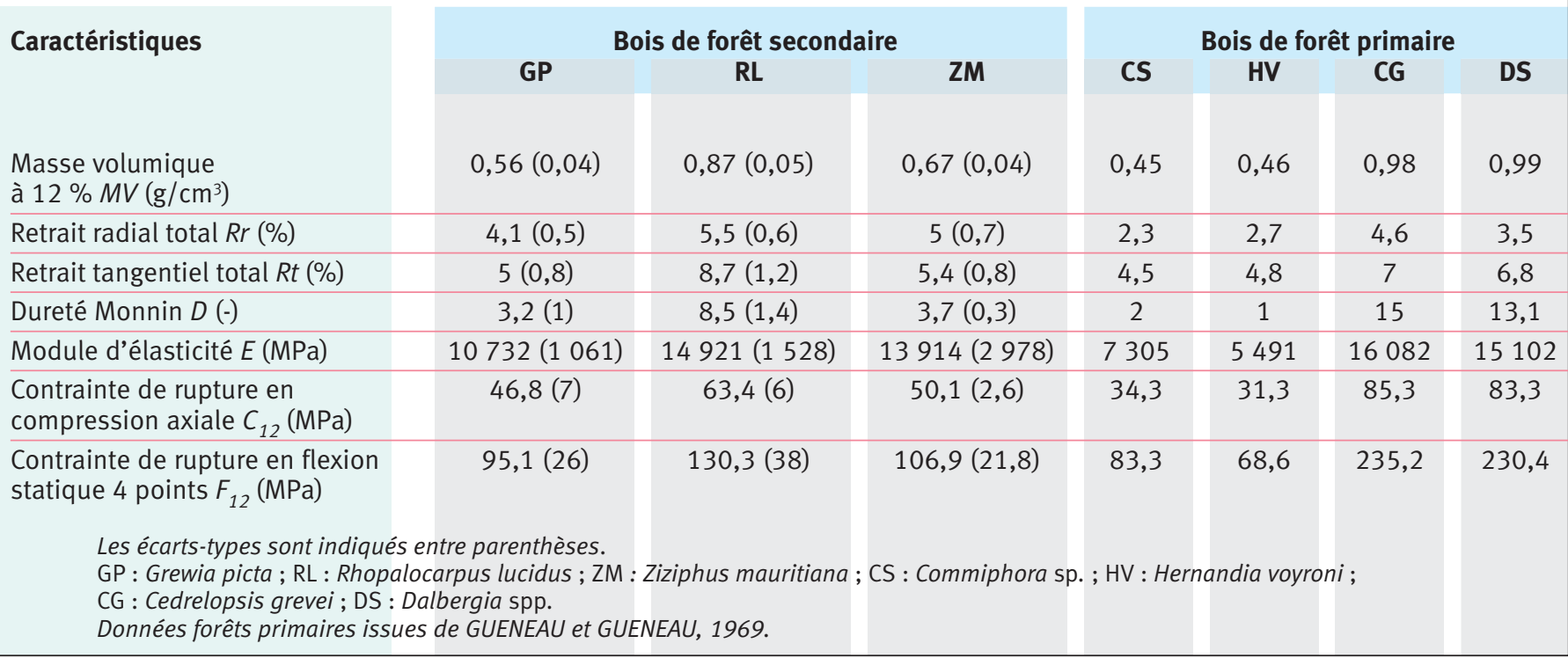




\section{Conclusion}

Différents produits et services sont tirés des ressources forestières aussi bien primaires que secondaires par les villageois. Les préférences sont dictées par les coutumes, par les connaissances empiriques des caractéristiques des ressources recherchées et par la disponibilité des ressources. De plus, les droits d'accès jouent également un grand rôle dans ce contexte. En effet, pour ce dernier point, les forêts primaires sont actuellement de plus en plus régies par des mesures généralement restrictives concernant la collecte. Dans certaines zones des aires protégées, même le passage est interdit. À titre palliatif, les forêts secondaires offrent une gamme de bois nécessaire à la vie quotidienne de la population mais leur valeur est encore mal connue. Parfois, le terme "secondaire » même disqualifie ces ressources forestières alors que la croissance des arbres y est plus forte, permettant une production de bois plus rapide que dans les forêts primaires. S'agissant des espèces utilisées par les villageois, des espèces de forêt secondaire viennent compléter les essences de valeur habituelles. Les caractérisations des propriétés physico-mécaniques en laboratoire ont confirmé le potentiel intéressant de ces bois de forêt secondaire, notamment des trois espèces les plus prisées par les villageois : Grewia picta, Rhopalocarpus lucidus et Ziziphus mauritiana. Cela reflète une capacité d'adaptation des villageois aux potentialités de la ressource forestière qui fournit les bois dont ils ont besoin. En effet, ils savent considérer, même de façon empirique, d'autres espèces qui sont relativement en abondance pour répondre à un besoin couvert jusqu'alors par une espèce devenue rare ou absente dans leur zone de prélèvement. Cette faculté d'adaptation des villageois mérite d'être prise en compte dans l'aménagement des paysages forestiers. Bien qu'il n'y ait encore aujourd'hui que peu d'expérience à travers le monde sur la gestion durable des forêts secondaires pour la production de matière ligneuse, la promotion d'espèces de substitution représente une option permettant de concilier la satisfaction des besoins en bois et la préservation de la biodiversité dans les forêts primaires. Les forêts secondaires constituent un recours potentiel pour la production rapide de ces espèces, dont l'accès est plus facile et oppose moins de contraintes aux villageois. Les forêts secondaires doivent donc désormais être prises en considération dans les politiques de gestion forestière au niveau national et international pour assurer leur durabilité mais aussi celle des forêts primaires.

Cette étude débouche sur d'autres recherches car il serait notamment intéressant de la compléter par la caractérisation des propriétés technologiques de l'ensemble des essences de forêts secondaires évoquées, que sont Terminalia fatrea, Strychnos decussata, Foetida retusa et Grewia cyclea. Par ailleurs, une étude plus approfondie sur les différences de choix d'espèces selon les villages, ethnies, âges et genre permettrait de mieux cibler les essences préférées dans chaque village et contribuerait à la possibilité d'extrapoler les résultats pour les localités ayant les mêmes critères d'utilisation du matériau bois.

\section{Remerciements}

Cette étude a été financée par le Groupe de foresterie pour le développement de l'École polytechnique fédérale de Zurich et la Fondation suisse Karl Popper. Les auteurs remercient les villageois du Menabe central pour leur contribution dans la réalisation de cette étude.

\section{Références bibliographiques}

ANDRIAMBELO L., 2005. Bilan des recherches en technologie du bois au sein du CFPF Morondava. Akon'ny Ala, 29 : 30-47.

ANDRIAMBELO L., 2010. Critères de gestion durable des ressources ligneuses du paysage forestier du Menabe central, Madagascar. Thèse, École supérieure des sciences agronomiques, Université d’Antananarivo, Madagascar, 167 p.

BORDONNÉ P. A., 1989. Module dynamique et frottement intérieur dans le bois. Mesures sur poutres flottantes en vibrations naturelles. Thèse, Inpl Nancy, France, 154 p.

BOURGEAT F., RANDRIAMBOAVONJY J. C., SOURDAT M., 1995. Les unités pédomorphologiques à Madagascar. Les facteurs de pédogénèse. Potentialités et contraintes régionales. Akon'ny Ala, 17 : 40-49.

CABALZAR G. P., 1996. Le milieu humain. In: Ganzhorn J. U., Sorg J.-P. (éds). Ecology and economy of a tropical dry forest in Madagascar. Primate Report, Special Issue, 46-1: 13-19.

CHAMBERS R., 1994. Participatory rural appraisal (PRA): challenges, potentials and paradigm. World Development, 22 (10): 1437-1454.

DE LAS SALAS G., 2002. Los bosques secundarios de América tropical: perspectivas para su manejo sostenible. Bois et Forêts des Tropiques, 272 (2): 63-73.

DGEF (DIRECTION GÉNÉRALE DES EAUX ET FORÊTS), 2003. Rapport national de Madagascar. In : La gestion des forêts tropicales secondaires en Afrique : Réalité et perspectives. Atelier Fao/EC Lnv/Gtz, Douala, Cameroun, 17-21 novembre 2003. Rome, Italie, Fao, 156-163.

DIRAC RAMOHAVELO C., 2009. Stratégies villageoises pour la gestion des paysages forestiers du Menabe Central, Madagascar. Thèse, École polytechnique fédérale de Lausanne, Suisse, $173 \mathrm{p}$.

EDWIN P., 2011. Investigation of processing characteristics of hardwood timbers from secondary forest in Papua New Guinea. Masters Research Thesis, Department of Forest and Ecosystem Science, Melbourne School of Land and Environment, The University of Melbourne, Australia, 396 p. 
EMRICH A., POKORNY B., SEPPTHE C., 2000. Significance of secondary forest management for development policy. Eschborn, Allemagne, Deutsche Gesellschaft für Technische Zusammenarbeit (Gtz), 93 p.

FAO, 2003. Vers l'aménagement durable et la mise en valeur des forêts secondaires tropicales en Afrique anglophone. La proposition d'action de Nairobi. Rome, Italie, Fao, Département Forêts, $20 \mathrm{p}$.

FAUROUX E., 2001. Dynamiques migratoires, tensions foncières et déforestation dans l'ouest malgache. In : Razanaka S., Grouzis M., Milleville P., Moizo B., Aubry C. (éds). Sociétés paysannes, transitions agraires et dynamiques écologiques dans le sud-ouest de Madagascar. Atelier Cnre-Ird, Antananarivo, Madagascar, 8-10 novembre 1999. Antananarivo, Madagascar, Cnre-Ird, 91-105.

FINEGAN B., 1992. The management potential of neotropical secondary lowland rain forest. Forest Ecology and Management, 47 (1-4): 295-321.

GRISA E., 1988. Anatomie ligneuse de 24 espèces feuillues de la côte ouest de Madagascar. Fiche Technique. Morondava, Madagascar, Centre de formation professionnelle forestière, $n^{\circ} 16,113 p$.

GUENEAU P., GUENEAU D., 1969. Propriétés physiques et mécaniques des bois malgaches. Nogent-sur-Marne, France, Centre technique forestier tropical, $76 \mathrm{p}$.

GUILLAUMET J.-L., KOECHLIN J., 1971. Contribution à la définition des types de végétation dans les régions tropicales (exemple de Madagascar). Candollea, 26 (2) : 263-277.

KOECHLIN J., GUILLAUMET J.-L., MORAT P., 1997. Flore et végétation de Madagascar. In : Tüxen R. (éd.). Flora et vegetatio mundi. Vaduz, Liechtenstein, A.R.G. Gantner, 701 p.

LUBINI A. C., 2003. Les aspects écologiques des forêts secondaires en Afrique centrale et occidentale francophone. In : La gestion des forêts tropicales secondaires en Afrique : Réalité et perspectives. Atelier Fao/EC Lnv/Gtz, Douala, Cameroun, 17-21 novembre 2003. Rome, Italie, Fao, 25-44.

MAEP, 2003. Monographie de la région du Menabe, unité de politique de développement rural. Morondava, Madagascar, Ministère de l'Agriculture, de l'Élevage et de la Pêche, 145 p. MEFT, USAID, Cl, 2009. Évolution de la couverture des forêts naturelles à Madagascar, 1990-2000-2005. 58 p.

MOAT J., SMITH P., 2007. Atlas of the Vegetation of Madagascar. Kew, Royaume-Uni, Royal Botanic Gardens, 780 p.

PAREDES A. A., 2011. Colombian lesser known hardwood species. Is there a potential for sustainable secondary forest management? In: International Scientific Conference on Hardwood Processing, 17 octobre 2011. Blacksburg, VA, États-Unis, Virginia Tech.

RAKOTOVAO G., RABEVOHITRA A. R., COLLAS DE CHATELPERRON P., GUIBAL D., GÉRARD J., 2012. Atlas des bois de Madagascar. Versailles, France, Éditions Quæ, 418 p.
RANDRIAMIDONA P., 1990. Les relations inter-ethniques dans le Menabe. Mémoire diplôme Ehess, Paris, France, $144 \mathrm{p}$.

RANDRIANASOLO J., RAKOTOVAO P., DELEPORTE P., RARIVOSON C., SORG J.-P., ROHNER U., 1996. Local tree species in the nursery. In: Ganzhorn J. U., Sorg J.-P. (éds). Ecology and economy of a tropical dry forest in Madagascar. Primate Report, Special Issue, 46-1: 117-132.

RATSINJOMANANA K., 2006. Impact de la structure socioculturelle sur la dynamique de la régénération naturelle de Hernandia voyroni (Capuron, 1966), Famille des Hernandiaceae. Cas de la forêt de Kirindy de Morondava. Université d'Antananarivo, École supérieure des sciences agronomiques, Madagascar, $62 \mathrm{p}$.

SHEIL D., PURI R. K., BASUKI I., VAN HEIST M., WAN M., LISWANTI N., RUKMIYATI, SARDJONO M. A., SAMSOEDIN I., SIDIYASA K., CHRISANDINI, PERMANA E., ANGI E. M., GATZWEILER F., JOHNSON B., WIJAYA A., 2004. À la découverte de la biodiversité, de l'environnement et des perspectives des populations locales dans les paysages forestiers. Méthodes pour une étude pluridisciplinaire du paysage. Jakarta, Indonésie, Center for International Forestry Research (Cifor), 101 p.

SORG J.-P., GANZHORN J. U., KAPPELER P. M., 2003. Forestry and Research in the Kirindy forest/Centre de Formation Professionnelle Forestière. In: Goodman S. M., Benstead J. P. (éds). The Natural History of Madagascar. Chicago, IL, ÉtatsUnis, University of Chicago Press, 1512-1519. 